\title{
Bacchus Neumonitis
}

Marta Sánchez-Marteles ${ }^{\mathrm{a}}$, María del Mar Rodero-Roldán ${ }^{\mathrm{a}}$, Mar Garcés-Sotillos $^{\mathrm{b}}$, Juan Ignacio Pérez-Calvo ${ }^{\mathrm{a}}$

a Internal Medicine Department, Hospital Clínico Universitario “Lozano Blesa”, Zaragoza, Spain

${ }^{\mathrm{b}}$ Allergy Department, Hospital Clínico Universitario “Lozano Blesa”, Zaragoza, Spain

\section{Abstract:}

Objective: Interstitial lung diseases (ILD) are a group of pathologies of undetermined frequency that require a broad differential diagnosis and continue to pose a challenge for clinicians.

Observations: We present a clinical case of a 17 -year-old male with acute interstitial pneumonitis, lung aspergillosis and foreign body lung granulomatosis after carbon monoxide (CO) intoxication. As far as we know, no similar cases have been reported in the literature.

Conclusions: ILD require a broad differential diagnosis, which is of great importance to prognosis.

Keywords: Pneumology, infectious diseases, internal medicine

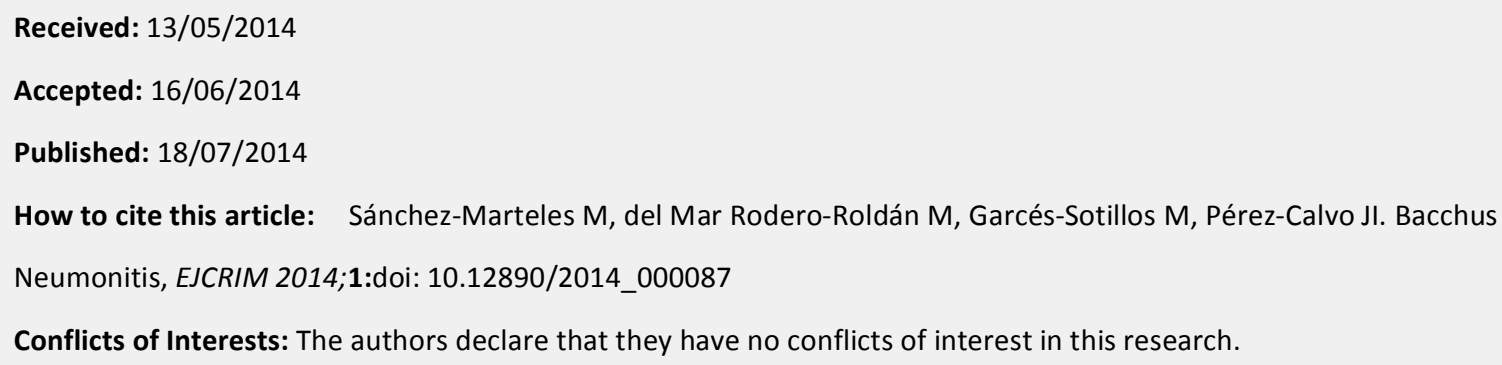

Conflicts of Interests: The authors declare that they have no conflicts of interest in this research. 


\section{Case report}

A 17-year-old male of Romanian origin and no relevant medical history was treated by emergency staff following an accident at work. He had fallen into a wine storage container several meters deep and had lost consciousness. On arrival at the emergency unit, he had severe respiratory failure and was transferred to the intensive care unit (ICU) with a presumptive diagnosis of acute non-cardiogenic pulmonary oedema. Carboxyhaemoglobin levels were normal.

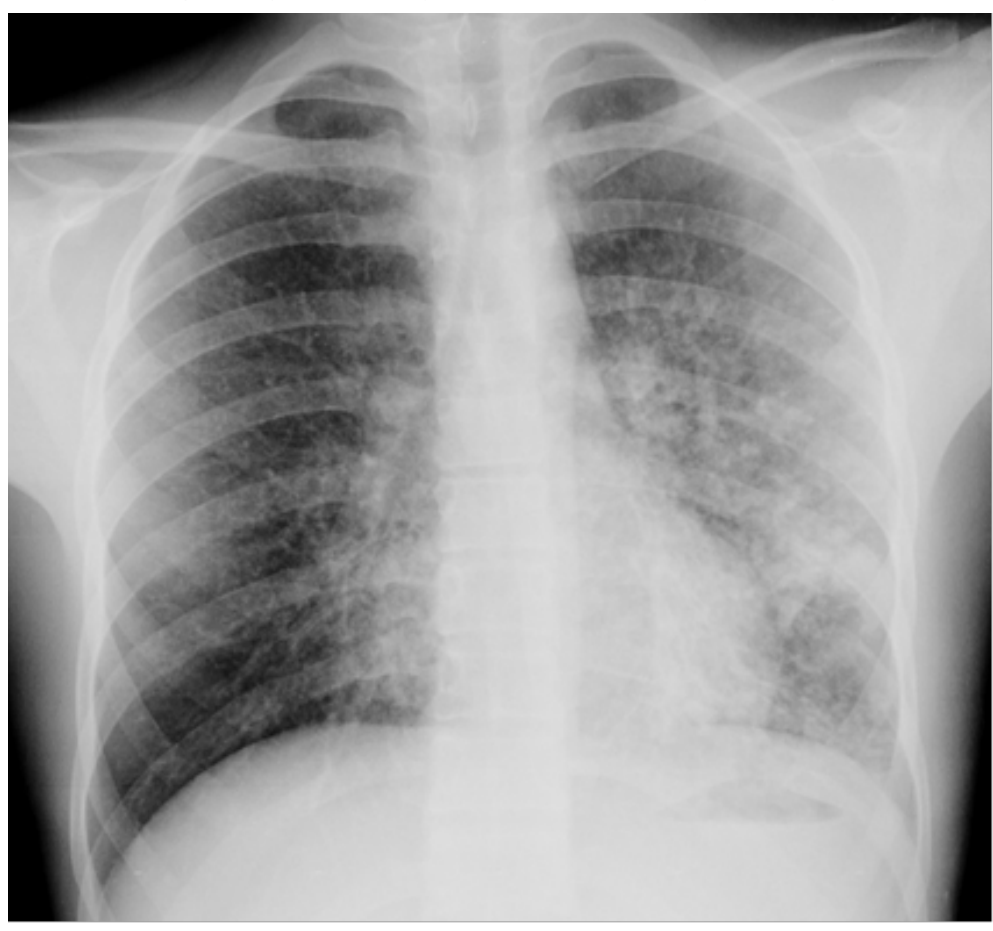

Figure 1: Chest $x$-ray showing an alveolar-interstitial pattern

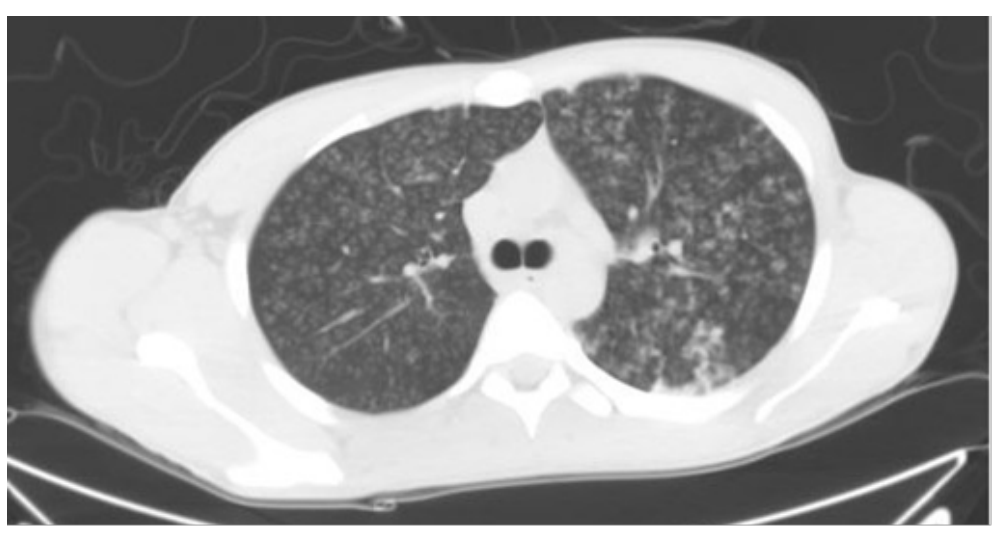

Figure 2: Well-defined centrilobular lung nodules affecting the entire pulmonary parenchyma, mainly in the right hemithorax
After stabilization in the ICU, the patient was transferred to the internal medicine ward with a diagnosis of carbon monoxide (CO) intoxication. He was febrile (up to $\left.39{ }^{\circ} \mathrm{C}\right)$, alert and pale. Tachycardia was 110 beats per minute (bpm) and jugular distension was not present. The respiratory rate was 28 per minute, accompanied by severe breathing difficulty. Crackling sounds could be heard from the middle and inferior lobes. The remainder of the physical examination was unremarkable. Partial oxygen pressure $\left(\mathrm{PO}_{2}\right)$ was 53 $\mathrm{mmHg}, \mathrm{PCO}_{2}$ was $41 \mathrm{mmHg}$, oxygen saturation was $88 \%$, and levels of bicarbonate and lactic acid were normal.

Chest x-ray showed an alveolar-interstitial pattern (Fig. 1). Microbiology of sputum, including investigation for Koch's bacilli, yielded negative results.

High-resolution thorax CT revealed well-defined centrilobular lung nodules 2-5 $\mathrm{mm}$ in diameter

covering the lung parenchyma, particularly in the right hemithorax. Lesions were attributed to hypersensitivity pneumonitis (Fig. 2). 


\section{European Journal \\ of Case Reports in \\ Internal Medicine}

Immunological tests were positive for IgG precipitins and Aspergillus fumigatus, and negative for autoantibodies (anti-centromere, anti-SSA/Ro, anti-DNAds, anti-RNP, anti-histone, anti-neutrophil cytoplasmic, anti-smooth muscle antibody).

There was no history of previous exposure to Aspergillus. The CD4/CD8 ratio was 1.52. Empirical treatment for tuberculosis (TB) was begun, followed by intravenous steroids administered according to the guidelines of the Spanish Pneumology and Thoracic Surgery Society (Sociedad Española de Neumología y Cirugía Torácica, SEPAR) for interstitial pneumopathies ${ }^{1}$. Treatment was well tolerated.

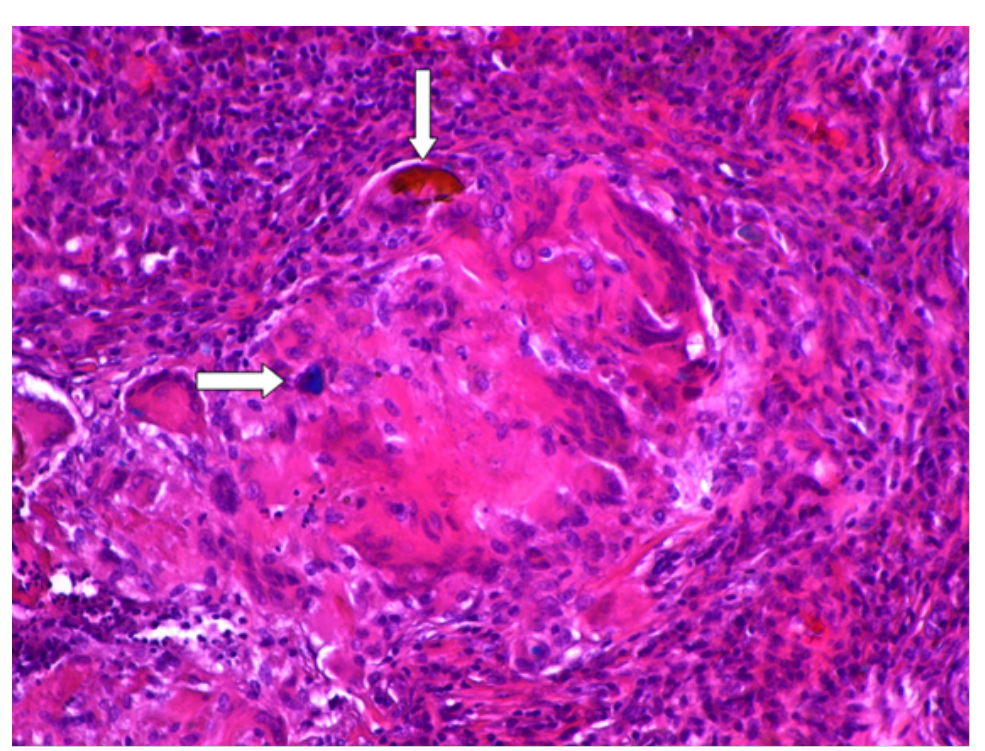

Figure 3: Foreign body giant cell granulomas (top arrow) and amorphous matter, refractive, yellow and blue (bottom arrow)
Respiratory function tests revealed a severe restrictive pattern. Bronchoscopy was contra-indicated due to severe respiratory failure. Consequently, an open lung biopsy was performed under anaesthesia. Pathological analysis of lung tissue showed a developmental-phase foreign body giant cell granuloma, without central necrosis There were ubiquitous deposits of amorphous, slightly fibrillar foreign bodies, which we identified as grape traces (Fig. 3).

Lung biopsy samples were cultivated, yielding a growth of $A$. fumigatus. PCR for mycobacterium complex was negative.

Anti-TB treatment was discontinued and voriconazole was added to corticosteroids, according to Spanish guidelines ${ }^{1,2}$.

The patient experienced a gradual clinical and radiological recovery, reaching NYHA functional class II and a respiratory in the British Medical Research Council (MCR) ${ }^{3}$. After discharge, respiratory function tests remained stable. It was significant that the CO diffusing capacity (DLCO) was $67.6 \%$ and the Tiffenau index 69.37 .

\section{Discussion}

Accidental CO poisoning is an occupational hazard of the wine fermentation process, frequently leading to a fatal outcome. Within minutes, inhalation of CO levels above $6-10 \%$ in air causes 
shortness of breath, dizziness, sweating, nausea and loss of consciousness. Treatment involves the administration of highly concentrated oxygen ${ }^{4}$.

Although broadly different in aetiology, diffuse ILD or interstitial pneumopathies are a group of diseases with similar clinical, radiological and functional features at presentation. The main differences allowing reliable classification are pathological changes involving the alveolar-interstitial structures. Such changes are associated with over 150 aetiological agents, although their epidemiology has not yet been clearly defined. From a clinical point of view, the most prominent symptom is dyspnoea during exertion or at rest, and cough. Other systemic signs such as fever, malaise, fatigue or arthromyalgia are also frequent. A wide range of diagnostic tests is required to rule out alternative diagnoses and correctly classify the subtype of ILD ${ }^{1,5,6}$.

One of the causes of ILD is hypersensitivity pneumonitis, which is caused by the inhalation of organic matter. Diagnosis is based on several criteria, such as provocation tests, detection of specific serum antibodies, and clinical improvement after eliminating exposure to the allergen. Our patient failed to fulfil any of these criteria ${ }^{1,5}$.

Most cases of pulmonary foreign body granulomatosis are associated with talc or cellulose used as excipients in medication or illicit drugs. To the best of our knowledge, there are no reported cases of ILD secondary to the foreign body reaction caused by the inhalation of grape traces ${ }^{7,8}$. The clinical pattern of our patient was similar to other cases of ILD. The most prominent symptoms were cough, sputum production and dyspnoea. There are fewer reported cases of ILD in which acute respiratory failure, fibrosis or emphysema have been caused by foreign body inhalation. Diagnostic work-up usually includes bronchoscopy and lung biopsy ${ }^{7}$. Treatment in the acute phase is based on general life-support measures, while in the chronic phase, the effectiveness of corticoids has not been proven, and the results of immunomodulators are based on animal models ${ }^{7,8}$.

We propose for this peculiar aetiology of ILD the name 'Bacchus pneumonitis', since it was caused by the full immersion of the patient in a deep wine storage container.

\section{Learning Points}

- The diagnostic work-up and treatment of interstitial lung diseases should be guided by the broad differential diagnosis of interstitial pneumopathies and a thorough and precise anamnesis.

- Early and precise diagnosis has significant effects on patients' quality of life and safety.

- The appropriate clinical approach includes the use of latest-generation technology, especially in such a complicated group of diseases. 


\section{References}

1. Xaubet A, Ancochea J, Blanquer R, Montero C, Morell F, Rodríguez Becerra E, et al. Grupo de Investigación en Enfermedades Pulmonares Intersticiales Difusas. Area de Técnicas y Transplante. SEPAR. Diagnóstico y tratamiento de las enfermedades pulmonares intersticiales difusas. Arch Bronconeumol 2003;39:580-600.

2. Walsh TJ, Anaissie EJ, Denning DW, Herbrecht R, Kontoyiannis DP, Marr KA, et al. Treatment of aspergillosis: clinical practice guidelines of the Infectious Diseases Society of America. Clin Infect Dis 2008;46:327-360.

3. Fletcher CM, Elmes PC, Fairbairn AS, Wood CH. The significance of respiratory symptoms and the diagnosis of chronic bronchitis in a working population. Br Med J 1959;1:257-266.

4. Viqueira JA. Toxicidades por oxígeno, monóxido de carbono y dióxido de carbono. JANO 2008;1707:23-30.

5. Wells AU, Hirani N, British Thoracic Society Interstitial Lung Disease Guideline Group. Interstitial lung disease guideline: the British Thoracic Society in collaboration with the Thoracic Society of Australia and New Zealand and the Irish Thoracic Society. Thorax 2008;63(Suppl. V):1-58.

6. Zacharisen MC, Fink JN. Hypersensitivity pneumonitis and related conditions in the work environment. Immunol Allergy Clin N Am 2011;31:769-786.

7. Conen D, Schilter D, Bubendorf L, Brutsche MH, Leuppi JD. Interstitial lung disease in an intravenous drug user. Respiration 2003;70:101-103.

8. Low SU, Nicol A. Talc induced granulomatosis. J Clin Pathol 2006;59:223-224. 